\title{
El estudio de las Relaciones Internacionales: Objeto, método, perspectivas
}

\section{Jean-Baptiste Duroselle*}

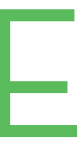

I creciente interés de muchos científicos sociales durante los últimos años en torno al estudio de las relaciones internacionales es un hecho que merece nuestra atención. En julio de 1950, Percy E. Corbett protestó en un artículo muy aclamado ${ }^{1}$ contra la tendencia de las universidades a negar al estudio de las Relaciones Internacionales un lugar autónomo dentro de las disciplinas estables, y colocarlo en los departamentos de Historia o Economía Política. A éstos podríamos añadir el departamento de Derecho, en el cual se enseña Derecho Internacional. William T. R. Fox, director adjunto del Instituto de Estudios Internacionales de la Universidad de Yale, ha demostrado amablemente ${ }^{2}$ que entre las dos guerras mundiales, los investigadores de Relaciones Internacionales se concentraron casi exclusivamente en el análisis de cuatro dominios bien delimitados donde la documentación era particularmente abundante: Derecho Internacional, organizaciones internacionales, relaciones comerciales y financieras e historia diplomática. "Ocasionalmente se hacía referencia a la importante contribución que los psicólogos, antropólogos y sociólogos podían hacer al estudio de las Relaciones Internacionales, pero rara vez se examinaba la naturaleza de esta contribución". Se hicieron esfuerzos, agrega el autor, por descubrir un mundo ideal, caracterizado por la paz permanente e universal; y no se describía el mundo real sino en referencia a este mundo ideal. En los últimos años, ha habido un progreso rápido en este sentido. Frederick S. Dunn, en su artículo destinado a dar seguimiento al de Flox, "The Present Course of International Relations Research"3, muestra muy bien que "los investigadores han desviado su atención del análisis descriptivo de las estructuras gubernamentales formales y lo han dirigido hacia una observación más cercana de los procesos políticos". Esta tendencia es particularmente fuerte en los Estados Unidos, donde numerosas universidades e instituciones han abordado esta tarea, como Yale, Harvard, la Biblioteca Hoover en la Universidad de Stanford, la Universidad de Washington, el Instituto de Estudios Avanzados en Princeton, la Institución Brookings, la Universidad de Michigan ${ }^{4}$ y la de Chicago, así como el esfuerzo de otras que han creado grupos de investigación de Relaciones Internacionales. A veces hay un "Comité de Relaciones Internacionales" en un "Departamento de

1 CORBETT, Percy E., "Objectivity in the Study of International Relations" en World Affairs, vol. 4, no 3, 1950, pp. 257-263.

2 FOX, William T. R., "Interwar International Relations Research: The American Experience" en World Politics, vol. 2, no 1, 1949, p. 77.

3 Ibídem, pp. 80-95.

4 Véase el programa de este en PASVOLSKY, Léo, "The Brookings Institution Program of International Studies" en World Politics, Vol. 2, no 2, 1950, pp. 295-303.
*Jean-Baptiste DUROSELLE (19171994), historiador, Doctor en Letras (1949), profesor en las Universidades de Sarrebrück y Lille (1950-1958), en el Instituto de Estudios Políticos de París (1946-1983) y en la Sorbona (desde 1970). Miembro de la Academia de Ciencias Morales y Políticas (desde 1975).

Este artículo ha sido traducido con el permiso de la editorial Revue française de science politique, Presses de Sciences Po (http://www.cairn. info/revue-francaisede-science-politique. htm). Publicado originalmente como DUROSELLE JeanBaptiste. "L'étude des relations internationales: objet, méthode, perspectives" en Revue française de science politique, $2^{\circ}$ año, n4, 1952, pp. 676-701, DOI: $10.3406 /$ Ifsp.1952.392166 http://www.persee. fr/doc/rfsp_00352950_1952

num_2_4_392166 [Consultado el 22 de enero de 2018]. (C) 1952, Presses de la Fondation Nationale des Sciences Politiques

Traducción: Diego Sebastián CRESCENTINO.

DOI:

http://dx.doi.org/ 10.15366/relacionesinternacionales2018.37.007 
Ciencias Políticas" dedicado a su estudio. Yo mismo participé, durante cuatro meses, en el "Comité de Relaciones Internacionales" de la Universidad de Notre Dame (Indiana) que, bajo el impulso del profesor Waldemar Gurian ${ }^{5}$, es uno de los más valiosos de esta Universidad. En Gran Bretaña, la London School of Economics, entre otras, ha creado cátedras sobre Relaciones Internacionales. En Francia, el Instituto de Estudios Políticos de París tiene, después de algunos años, una sección especial de Relaciones Internacionales ${ }^{6}$. Alemania parece estar mucho menos avanzada en esta dirección, pero hay indicios de que se están produciendo avances ${ }^{7}$.

Existe, por lo tanto, una tendencia muy general a abordar el estudio de las Relaciones Internacionales como una disciplina autónoma. Esto se explica por la conciencia cada vez más clara que tienen los investigadores de la existencia de un conjunto de fenómenos específicos, que merecen ser objeto de un estudio particular. Este conjunto de fenómenos es bastante fácil de categorizar. Todo lo que se refiere a las relaciones de un estado con otro estado, o de varios estados entre sí en el plano político, económico, social, demográfico, cultural o psicológico, puede ser situado allí; y aún más general, todo aquello que aborde las relaciones entre diferentes grupos a ambos lados de las fronteras nacionales puede ser contemplado a partir de esta disciplina. Si de lo que se trata es de las relaciones entre estados, podemos llamarle "política exterior". En cambio, si de lo que se trata es de relaciones entre grupos, podemos hablar de "vida internacional". Todos estos fenómenos constituyen las "Relaciones Internacionales".

Estudiar científicamente el conjunto de fenómenos que constituyen las Relaciones Internacionales es, a priori, posible. El único criterio del valor de tal estudio es el logro de resultados apreciables. No obstante, abordaremos este problema más adelante. Anteriormente, es importante comprender que dicho estudio se distingue necesariamente de las disciplinas más antiguas con las cuales, hasta los últimos años, habían estado envueltas las Relaciones Internacionales: $1^{\circ}$ del derecho internacional, porque éste especula sobre lo que debe ser, mientras que aquellas estudian lo que es; se trata entonces de la misma diferencia existente entre el Derecho y la Sociología; $2^{\circ}$ de la historia diplomática, porque su objetivo es estudiar los fenómenos del pasado y explicar su evolución, mientras que las primeras se caracterizan por el estudio de fenómenos actuales; y 30 de economía política, porque ésta solo aborda un aspecto de las Relaciones Internacionales. La cuestión de si el estudio de las Relaciones Internacionales es una rama de la Ciencia Política, o bien si constituye una disciplina separada, me parece puramente académica. Toda respuesta a esta cuestión depende de la definición de Ciencia Política que se contemple.

\footnotetext{
5 GURIAN, Waldemar, "On the study of International Relations" en The Review of Politics, vol. 8, no 3, 1946, pp. 275-282.

6 Véase el libro publicado por la UNESCO: La Science politique conemporaine, Paris, 1950, y en particular el artículo de Pierre Renouvin, "La Contribution de la France à l'étude des relations internationales", pp. 578-592. Acerca de la enseñanza - que yo no abordo aquí, limitándome a la investigación-, Jacques Chapsal, director del Instituto de Estudios Políticos de Paris, hizo una presentación en el Congreso de Winsor en diciembre de 1950, titulada: Note sur l'enseignement des relations internationales en France; el texto, traducido al inglés, fue publicado en las actas de este congreso: The University Teaching of International Relations, editado por G.L. Goodwin, Oxford-Paris, 1951, pp. 84-94.

7 Véase el importante artículo de MEYER, Ernst Wilhelm, "Politische Wissenschaft und Außenpolitik" en Außenpolitik, febrero 1952, pp. 73-80: defensa para que admitamos la existencia de un estudio autónomo de las Relaciones Internacionales.
} 
Tras admitir la existencia de este conjunto de fenómenos especiales que constituyen las "Relaciones Internacionales", el propósito de este artículo es examinar en qué consisten y a través de qué método deben ser abordadas. Naturalmente, existe ya una considerable cantidad de trabajos sobre Relaciones Internacionales, y no pretendo establecer reglas metodológicas absolutas para una línea de pensamiento previo. En su lugar, propongo hacer un trabajo de síntesis basado en experiencias pasadas. No pretendo con ello ocultar el carácter provisional e incompleto de mi objetivo, pero sin duda sería útil hacer un balance y elaborar una especie de evaluación, aunque solo sea para iniciar una discusión fructífera. A esta presentación, añadiré finalmente algunos puntos de vista sobre las perspectivas disponibles en Francia en torno a esta área.

1.

Tratemos en primer lugar de tener una visión general de lo que debería ser el estudio de las Relaciones Internacionales. Una primera observación es necesaria: no se trata estrictamente hablando de un "arte" o de una "ciencia pura". Considerarlo como un arte sería admitir que conocemos las Relaciones Internacionales únicamente a través de un espíritu de astucia, de intuición, de una especie de don. Hay buenos y malos diplomáticos, algunos dotados y otros no. Pero no debemos exagerar el alcance de esta observación y creer que con contar con este don es suficiente, ya que esto conllevaría a que el conocimiento profundo de estas cuestiones sería superfluo ${ }^{8}$. Aristide Briand sólo podía fiarse de su notable intuición y de su conocimiento instintivo de los individuos porque tenía detrás de sí a Philippe Berthelot, quien tenía conocimiento de los registros ${ }^{9}$. Por otra parte, la diplomacia es una actividad profesional, mientras que el estudio de las Relaciones Internacionales solo puede ser trabajo de un investigador. El diplomático solo las usa como una ciencia aplicada. Se trata de la misma diferencia que existe entre el científico de laboratorio y el ingeniero. Sería absurdo decir que la intuición es suficiente para conocer la gran cantidad de hechos y reacciones que constituyen las Relaciones Internacionales.

Pero en el sentido opuesto, hacer del estudio de esta disciplina una "ciencia pura" sería una exageración análoga ${ }^{10}$. El carácter propio de una ciencia es que conduce a leyes, es decir, a la posibilidad de predecir. Sin embargo, no hay razón para afirmar que una vez que la madeja de fenómenos internacionales hayan sido mejor estudiados, llegaremos a la formulación de leyes seguras que permitan predecir fenómenos y actuar con certeza. Sin duda, este es un rasgo común a todo el orden de conocimiento agrupado bajo el nombre de "ciencias sociales". Desde el momento en que aparece la mente humana, todo sucede como si la indeterminación entrara en juego. Admitir que la mente humana, tomada individualmente o en grupos, está sujeta a leyes rigurosas, es plantear una premisa de la cual, hasta ahora, nada se ha podido verificar, incluso en las Ciencias Sociales más avanzadas, como la Economía política o la Historia. Por lo tanto, establecer que el objetivo del estudio de las Relaciones Internacionales es el descubrimiento de leyes exactas que permitan predecir el futuro con certeza, parece guiar esta disciplina en una dirección engañosa y condenar a los investigadores a realizar

\footnotetext{
8 Véase MEYER, E.W., op. cit. en Außenpolitik, 1952, pp. 77-78.

9 Véase también en torno a este tema el trabajo de BREAL, Philippe Berthelot, p. 206.

${ }^{10}$ Véase los interesantes comentarios de MURDOCK, George Peter, "The Conceptual Basis of Are Research" en World Politics, vol. 2, no 4, 1950, pp. 571-578.
} 
enormes esfuerzos para obtener resultados decepcionantes ${ }^{11}$.

¿Significa esto que el estudio de las Relaciones Internacionales como "ciencia social" y no como "ciencia pura" debe ser simplemente descriptivo, es decir, una inminente acumulación de información en la cual renunciaríamos a conseguir nada? Ciertamente no. Está claro que tal estudio puede derivarse de lo que llamaré "datos fundamentales" que no son leyes en el sentido de que no necesariamente determinan la "política exterior" del estado, del grupo de estados considerados, o la "vida internacional" de uno o más grupos de individuos; pero sí indican cómo esta política exterior o esta vida internacional estarán probablemente orientadas. Estos "datos fundamentales" pueden explicarse por factores muy complejos, algunos permanentes como la geografía física, otros duraderos como la organización social. El propósito del estudio de las Relaciones Internacionales debe ser antes que nada descubrir, en la masa inextricable de los fenómenos, cuáles son estos "datos fundamentales" y liberarlos de los "datos accidentales" cuya influencia puede ser inmensa pero sigue siendo transitoria. ¿Hasta qué punto las relaciones entre Roma y el Egipto ptolemaico se explican por causas geográficas, económicas, sociales y políticas, profundas y duraderas, o por la nariz de Cleopatra?

Poder discernir entre datos fundamentales y duraderos y datos accidentales y efímeros; tal debe ser el objetivo del estudio de las Relaciones Internacionales. Por ejemplo, es sorprendente que en la política exterior francesa haya habido durante siglos un conflicto entre una política continental de expansión o de seguridad y una política colonial, con profundas raíces comerciales. Es el conflicto entre Louis XIV y Colbert, es la Guerra de los Siete Años donde Francia pierde India y Canadá debido a su lucha contra Prusia, es la conquista de Argelia para confortar la opinión de los tratados de 1815, es la política de la "Liga Azul de los Vosgos" y de la revancha en oposición a la expansión colonial y al acercamiento con Alemania. Aún hoy, es claro que el dilema existe y que el número de divisiones que Francia puede proporcionar a la O.T.A.N. está limitado por la guerra de Indochina. ¿No es este conflicto un dato fundamental y duradero de los franceses?

En Gran Bretaña, por el contrario, tanto los laboristas como los conservadores tienen una tendencia insuperable a colocar los problemas de la Mancomunidad de Naciones por sobre los de Europa, y se niegan a ceder una parte de su soberanía para mantener las manos libres en sus relaciones al interior de la Mancomunidad. ¿No sucedió esto al final de la era victoriana, cuando el imperialismo iba de la mano con el "espléndido aislamiento", y durante la Guerra de los Siete Años, cuando la defensa de Hannover se consideró infinitamente menos importante que la de las posesiones de ultramar? Estos son casos relativamente simples, y pueden ser multiplicados con otros ejemplos en Rusia, Estados Unidos, Alemania. Desde el campo de la política exterior hasta el de la vida internacional, uno podría ciertamente descubrir datos fundamentales en el estudio de la emigración italiana, la inmigración de los Estados Unidos o de Australia. Además de estos datos de sencillo acceso, hay muchas razones para creer que existen otros tan importantes como estos, y que solo los descubriremos tras efectuar un análisis detallado de los fenómenos internacionales. A pesar de sus aparentes

\footnotetext{
${ }^{11}$ No comparto sobre este punto las ideas de GUETZKOW, Harold, "Long Range Research in International Relations" en American Perspective, vol. 4, no 4, 1950, pp. 421-440.
} 
contradicciones, obras como las de Charles A. Beard ${ }^{12}$ o Hans Morgenthau ${ }^{13}$ sobre los fundamentos de la política exterior estadounidense constituyen buenos ejemplos de análisis de los hechos, que llevan a conclusiones científicas sin dejarse influir por una visión ideal del mundo. Su trabajo sólo comienza a ser discutible en el momento en el cual se vuelven normativos, es decir, cuando exponen lo que debería ser la política exterior estadounidense ${ }^{14}$. Las conclusiones científicas que alcanzan, como el poder de la corriente aislacionista en los Estados Unidos magistralmente descrito por Beard ${ }^{15}$, o la orientación hacia una política de esferas de influencia, indicada por Morgenthau, no son irreconciliables, e incluso marcan etapas. El análisis de Klingsberg, que identifica períodos de "introversión" y "extroversión" en la política exterior estadounidense, podría servir a modo de síntesis de sus conclusiones ${ }^{16}$. Estas diversas obras, dado el estado todavía embrionario del estudio de las Relaciones Internacionales y la insuficiencia de trabajos de base, siguen siendo muy discutibles, pero muestran claramente que hemos dejado la fase empírica de investigación para entrar en una fase científica, y su contribución al descubrimiento de ciertos datos fundamentales de la política exterior estadounidense está lejos de ser insignificante.

Despejar los datos fundamentales y los datos accidentales implica que no nos limitamos a estudiar situaciones, sino que también nos ocupamos de movimientos. Sin duda, como ya he dicho, el estudio de las Relaciones Internacionales es el de los fenómenos actuales. Pero estos fenómenos actuales no pueden explicarse si están separados artificialmente de la evolución en la que se encuentran. Por ejemplo, la existencia de un grupo comunista en Francia tiene una influencia determinada, en una fecha determinada, en la política exterior de Francia, en relación con su importancia relativa en la nación. Pero la situación es bastante diferente dependiendo de si esta importancia relativa ( $25 \%$ de los votantes, por ejemplo) está aumentando visiblemente o disminuyendo visiblemente. Para saber si está aumentando o disminuyendo, debemos estudiar la historia de este grupo comunista y así ubicar el fenómeno actual -su influencia en el momento en que estamos- en el movimiento. Cuanto más preciso sea el estudio del contexto histórico, más válida será la idea del fenómeno actual. Por otro lado, el hecho de que la intensidad de una influencia aumente o disminuya no debería llevar a una extrapolación imprudente, según la cual el crecimiento o la decadencia se considerarían permanentes. Puede haber fases, regulares o irregulares, de crecimiento y descomposición. En relación a la población de los Estados Unidos, es bien sabido que, extrapolando los fenómenos contemporáneos, los demógrafos estadounidenses de la década de 1930 concluyeron que la población estadounidense se estabilizaría en la década de 1950.

\footnotetext{
12 Por ejemplo BEARD, Charles A., American Foreign Policy in the Making 1932-1940: A Study in Responsibilities, Yale University Press, New Heaven, 1946.

13 MORGENTHAU, Hans J., In defense of the national interest: A critical examination of American foreign policy, Alfred A. Knopf, Nueva York, 1951.

${ }^{14}$ Beard ha sido a menudo criticado en este punto, pero su método es el de un precursor: Véase lo que dice W. Fox (op. cit., p. 77): "Ya había escrito en 1930: Quiero saber qué diablos es realmente. Beard alcanzó lo que muchos considerarían como tener las respuestas incorrectas es menos importante que hacer las preguntas correctas". En cuanto a Morgenthau, en un informe reciente ha sido criticado por abandonar la actitud científica y adoptar una actitud normativa: TUCKER, Robert W., "Professor Morgenthau's Theory of Political Realism" en American Political Science Review, vol. 46, no 1, 1952, pp. 214-224.

${ }^{15}$ Véase también lo que dice Morgenthau sobre un "neo-aislacionismo" de diferente carácter pero de origen idéntico. En In defense of National Interest, op. cit., pp. 128-138.

${ }^{16}$ BEARD, Charles A. "The Historical Alternation of Moods in American Foreign Policy" en World Politics, vol. 4, no 2, 1952, pp. 239-273.
} 
Sin embargo, las tendencias se han revertido y, como sabemos, la población estadounidense ha comenzado a crecer más rápidamente. Un fenómeno similar se ha observado en Francia.

Por lo tanto, el estudio de las Relaciones Internacionales solo obtendrá resultados válidos si acude a la Historia. La influencia que ejerce la composición de los grupos sociales de un país en su política exterior no solo requiere una investigación sociológica exhaustiva que refleje la situación actual, sino también un estudio de la historia social que muestre cuál es el significado de la evolución de los diversos grupos sociales considerados. Del mismo modo, la comprensión de las relaciones económicas internacionales no solo requiere un análisis económico del fenómeno actual, sino un estudio de la historia económica que muestre en qué momento se encuentra este fenómeno. Los ejemplos son múltiples. Todos demostrarían que la perspectiva histórica es indispensable en todos los casos, aunque solo sea para determinar, entre los fenómenos, aquellos que son continuos y aquellos que son radicalmente nuevos. En la comunicación entre los pueblos, aspecto capital de la vida internacional, el surgimiento del cine mudo, el cine sonoro, la radio y la televisión son fenómenos nuevos e introducen nuevos "datos" que es importante reconocer, unos fundamentales y otros accidentales.

\section{2.}

Ya contamos con una lectura general de aquello que constituye la disciplina de las Relaciones Internacionales: el estudio científico de los fenómenos internacionales para conseguir distinguir entre los datos fundamentales y accidentales que los rigen. ¿Cuáles son estos fenómenos internacionales que constituyen la materia, el objeto de estudio? Podemos dar una definición general y decir que un fenómeno es internacional tan pronto como implique cualquier relación a ambos lados de una frontera nacional. Pero esta definición es demasiado general para ser útil. Parece más interesante elaborar empíricamente una lista de las preguntas que deben ser formuladas para tener una idea lo más completa posible de los fenómenos internacionales y para revelar los datos que los regulan. Las Relaciones Internacionales son principalmente relaciones políticas de estado a estado, y luego relaciones grupales o individuales a ambos lados de una frontera. Enfatizaré el primer aspecto, que es más accesible: la política exterior ${ }^{17}$.

\section{1. ¿Quién elabora la política exterior?}

Este problema merece ser estudiado considerando cada estado. Las condiciones son, de hecho, muy diferentes y variables según los tiempos. En la Alemania bismarckiana está claro que el Canciller fue el autor de todas las decisiones importantes, y la influencia de Guillermo I fue secundaria, como lo demuestra la conclusión de la alianza austro-alemana de 1879, dirigida contra Rusia a pesar de la voluntad expresa del emperador. En la Alemania de Guillermo II, en ciertas épocas no está claro a primera vista si las decisiones son tomadas por el Emperador, el Canciller, el Secretario de Estado de Asuntos Exteriores o "la eminencia gris", el Barón de Holstein. En Francia, las acciones del Ministro de Asuntos Exteriores y del Presidente del Consejo no siempre están claramente definidas y, a veces, hay conflictos, como lo demuestran las dificultades de 1905 (Delcasse - Rouvier) o 1911 (de Selves - Caillaux). En

\footnotetext{
17 Hay una guía muy valiosa sobre este tema: KURT, London, How Foreign Policy is Made? Van Nostrand Political Science Series, Nueva York, 1949. A menudo me referiré a ella siguiendo un plan que me parece más lógico. Por otro lado, me refiero aquí a la primera parte, Elements of power politics, del hermoso libro de SCHWARZENBERGER, Georg, Power Politics: A Study of International Society, Frederick A. Praeger, Nueva York, 1951 pp. 25-260 [1ra edición de 1949].
} 
general, es el Consejo de Ministros quien arbitra tales disputas y toma las decisiones supremas. En los Estados Unidos, el Secretario de Estado siempre está estrechamente subordinado al Presidente. Estos ejemplos simples muestran que el problema no es insignificante y que es necesario realizar un estudio preciso.

\section{2. ¿Qué influencias sufren los autores de la política exterior? ${ }^{18}$}

Este es un problema extremadamente complejo y poco entendido. Es cierto que el autor de una política exterior está estrechamente influido, como cualquier persona de acción, por el medio social al cual pertenece y por su educación. Pero él está además bajo una presión más directa. Tiene consejeros cuya influencia exacta podrá determinarse según el grado de confianza que tiene en ellos. Harry Hopkins tuvo mayor influencia con Roosevelt que el almirante Leahy, por ejemplo. En cada problema, hay equipos de personas que trabajan e intentan hacer prevalecer sus ideas. Por ejemplo, en Gran Bretaña, el equipo de Oriente Medio, con el General Spears, fue violentamente antifrancés en 1945, mientras que unos meses más tarde, el equipo del sudeste asiático, con el almirante Louis Mountbatten, apoyó enérgicamente la política francesa en Cochinchina.

Más allá de los equipos y las oficinas, existe la influencia fuerte, compleja y difícilmente comprensible de la opinión pública y, junto con ella, de la política interna. Obviamente, estas influencias dependen del régimen político. Si es totalitario, el régimen puede descuidar la presión de la opinión pública o moldearla a través de medios poderosos. Si es democrático, debe tenerlos en cuenta. Pero la opinión pública es el resultado, a veces difícil de comprender, de las actitudes a menudo contradictorias de grupos variados. ¿Hasta qué punto expresan los periódicos esta situación? Es bien sabido que a menudo estos son respaldados por grupos financieros o potencias extranjeras, que buscan influir en la opinión pública o dar una falsa idea de ella a los dirigentes. En raros casos sucede, sin embargo, que la opinión pública impone su forma de ver las cosas. Tal fue la situación durante diciembre de 1935 en Gran Bretaña, cuando, indignada por la revelación de un plan de partición de Etiopía llamado el "Plan Laval-Hoare", la opinión pública obligó a Sir Samuel Hoare, jefe de la Oficina de Relaciones Exteriores, a renunciar. Es sin duda durante el período 1933-1941 en los Estados Unidos donde esta influencia ha sido mejor estudiada. El trabajo de Charles Beard muestra cómo la opinión pública forzó a Roosevelt a practicar una política aislacionista. El reciente trabajo de Langer y Gleason: The Challenge to Isolation ${ }^{19}$ muestra como Roosevelt tuvo en cuenta la opinión pública estadounidense en los años cruciales de 1937-1940, parcialmente orientándola, parcialmente librándose de ella.

Por supuesto, la influencia de los parlamentos y asambleas electos es considerable; pero no siempre es fácil de analizar, y también requiere una extensa investigación para el caso de cada país.

Se plantean asimismo otras presiones, públicas u ocultas: grupos de interés, sindicatos patronales y sociedades financieras, sindicatos obreros, asociaciones patrióticas, iglesias, sociedades de pensamiento. Esta red solo puede comprenderse al comparar, valiéndonos de

\footnotetext{
${ }^{18}$ Sobre este tema, Renouvin prepara un libro que puede servir como base para futuras investigaciones.

${ }^{19}$ LANGER, William L. y GLEASON, S. Everett, The Challenge to Isolation, 1937-1940, Harper, Nueva York, 1952.
} 
documentos, las decisiones tomadas por estos grupos y sus tomas de posición. Más aún, es preciso saber si ha habido contacto directo entre el ministro y los representantes de estos grupos. También hay que considerar el carácter más o menos influenciable de la persona a cargo. Está claro que el mariscal Petain, en 1940, siguió a su vez las sugerencias de Paul Baudouin, favorable al establecimiento de estrechas relaciones secretas con Gran Bretaña, y Pierre Laval, favorable a la política de colaboración. Es curioso observar que su decisión de reunirse con Hitler en Montoire el 24 de octubre sucede tras una grave enfermedad de Baudouin.

Del mismo modo es necesario considerar no solo las teorías personales de los líderes, sino también el espíritu más o menos realista que muestran. En noviembre de 1923, Poincaré aceptó una solución internacional del problema del Ruhr por razones económicas, pero también por razones relacionadas con su espíritu legal, lo que le llevó a preferir el regreso al sistema de la "Comisión de reparaciones" creada por el Tratado de Versalles en lugar de seguir adelante con una política de fuerza.

La realidad política, económica y social impone un marco en el cual la iniciativa del hombre de estado no es ilimitado (ver cita número 5 ).

\section{3. ¿Quién ejecuta la política extranjera?}

En esta área, como en muchas otras, es necesario considerar cuidadosamente a las agencias ejecutoras, ya que existen a menudo distorsiones entre las directivas asignadas y las llevadas a cabo en la etapa de implementación. Esencialmente, el órgano de ejecución está constituido por el cuerpo diplomático. También pueden estar involucrados otros cuerpos de funcionarios en las relaciones económicas y culturales. En algunos países, como Países Bajos, hay dos administraciones separadas: una compuesta por un cuerpo de funcionarios que hayan hecho sus carreras en el Ministerio de Relaciones Exteriores, y la otra compuesta por diplomáticos. Este sistema, aplicado en los Estados Unidos, fue duramente criticado ${ }^{20}$. Ello se debe a que la administración central tendía a ignorar a los países extranjeros y diplomáticos que no tenían suficientemente en cuenta la situación política interna de su propio país. En la mayoría de los estados, como es el caso de Francia, hay un solo organismo con funcionarios que alternan entre la administración central, el "Departamento", y los puestos en el extranjero. Sea como fuere, es imperativo un estudio sociológico del cuerpo diplomático: su composición social, su formación y sus tradiciones desempeñan un gran papel. La Alemania nazi conservó a muchos diplomáticos de carrera cuya política personal estaba a menudo en desacuerdo con la del Führer. Así, von Schulenburg, el embajador en Moscú, realizó esfuerzos sobrehumanos para evitar la ruptura de su país con la Unión Soviética en 194121. En Francia, la contratación desde 1946 del cuerpo diplomático por parte de la Escuela Nacional de Administración, no dejará de producir, a largo plazo, cambios significativos en comparación con el antiguo sistema del "gran" y el "pequeño concurso".

${ }^{20}$ Véase el curso profesado por la Sra. Vera Dean en el Instituto de Estudios Políticos de la Universidad de París en 1948. La autora mostró que el informe Hoover abogaba por la fusión de los dos cuerpos.

${ }^{21}$ Véase el compendio Nazi-Soviet Relations. En este punto, tomo en cuenta una conferencia dada en la Sociedad de Estudios Alemanes en 1952 por Maurice Baumont. 


\section{4. ¿Cuáles son los medios de información de las autoridades responsables?}

Esto ciertamente merece un análisis detallado. Existe una fuente tradicional de información: los informes de los agentes diplomáticos. Pero en los estados modernos, los líderes toman cada vez más en cuenta la información proporcionada por el servicio o los servicios de inteligencia. Éstos reflejan a veces la realidad con mayor fidelidad que los diplomáticos, pero tienen la desventaja de ser difíciles de controlar y presentan una tendencia a "dramatizar" las situaciones. No hay dudas de que Hitler, y especialmente Mussolini - un ex periodista con un buen conocimiento de las tecnologías de la información-, hicieron un uso poco moderado, casi exclusivo de los servicios secretos de inteligencia y tomaron muchas decisiones basadas en sus informes. Cualquier fuente de información, diplomática, militar, policial, etc. tiene un defecto grave en el caso de los gobiernos dictatoriales: los agentes buscan a menudo proporcionar información que complazca a la autoridad, y tienen por lo tanto una cierta tendencia a distorsionar la realidad. La dificultad para los investigadores de acceder a los archivos del servicio secreto no debe frenar los intentos de resolver este delicado problema.

\section{5. ¿Cuáles son los medios de acción?}

Obviamente, esto está relacionado con el poderío de cada país en cuestión. Pero, ¿qué es exactamente el poderío? Si bien hoy podemos identificar dos potencias centrales, es difícil determinar el grado de poderío de los otros países. Schwarzenberger ha realizado un análisis detallado de esta noción ${ }^{22}$ y ha demostrado que lo que caracteriza a una gran potencia no es su extensión ${ }^{23}$ ni su población, sino su "fuerza", es decir, su número de divisiones, de aeronaves o navíos, su potencial industrial, su capacidad intelectual y su cohesión moral. Naturalmente, la noción de potencial industrial incluye la de recursos en materias primas, lo que casi inevitablemente implica un área metropolitana o colonial bastante grande. Por otro lado, el país debe poseer una gran autonomía en materia económica, es decir, ser lo suficientemente rico como para enfrentar los enormes costos que supone una guerra moderna.

Finalmente, añadiremos que los medios de acción de una potencia están limitados por ciertas características geográficas ${ }^{24}$; la inmensidad de Rusia y la extensión de sus comunicaciones aumentan la capacidad defensiva y probablemente dañan la capacidad ofensiva soviética. La extensión de la costa de los Estados Unidos obliga a este país a ser una gran potencia naval. Los ejemplos a tener en cuenta son múltiples.

\section{6. ¿Cuáles son los métodos de acción?}

Para responder a esta pregunta, es necesario analizar las técnicas utilizadas en relación con cada hecho y cada evento por los estadistas y diplomáticos, por los autores de las decisiones y por los ejecutores. Su variedad es extrema. Gracias a numerosos documentos, memorias y obras de Derecho internacional, conocemos bien tanto las técnicas antiguas y tradicionales tales como la negociación diplomática o el encuentro entre dos jefes de gobierno o ministros-; como las técnicas nuevas, o al menos renovadas -como las conferencias internacionales o el empleo de organizaciones internacionales-. Pero la investigación en torno a estos métodos

\footnotetext{
22 SCHWARZENBERGER, Georg, "Towards the Climax" en World Affairs, octubre de 1950, pp. 385-400.

23 J. Gottmann, en su libro La politique des Etats et leur géographie (Paris, A. Colin) insiste en que la contigüidad de los territorios es incluso más importante que su extensión.

${ }^{24}$ Véase sobre este tema el libro de Gottmann citado anteriormente y, del mismo autor: GOTTMANN, Jean, "Geography and International Relations" en World Politics, vol. 3, nº 2, 1951, pp. 153-173.
} 
formales no debe olvidar el análisis de los medios de presión presentes en ellos. En este sentido, es necesario estudiar la presión moral infligida en nombre del Derecho internacional, asumida en cierto modo por una determinada opinión pública internacional cuyo desarrollo actual es confuso. A esta presión moral se añade la posible amenaza directa ejercida por el gobierno demandante, o aquella indirecta ejercida por las organizaciones de medios de comunicación. Así actuó Hitler en 1938 y 1939. La mera existencia de esta amenaza conlleva a que las técnicas de respuesta a ellas deben ser estudiadas: apaciguamiento o "endurecimiento", contención o "contraofensiva". Nos guste o no, debemos reconocer que los estadistas han perfeccionado estas técnicas desde 1933.

Ahora bien, más allá de la amenaza, se puede usar la presión por la fuerza, lo cual supone o no una guerra según la cual el país sometido a la presión resiste o cede.

Finalmente, existen métodos de acción relativamente nuevos vinculados a descubrimientos científicos además de los procesos diplomáticos y militares, a cuyo fin se destinan cada vez mayores sumas presupuestarias en diversos países. Se trata de las técnicas de propaganda internacional. Esto casi siempre se hace fuera del accionar de los diplomáticos, quienes la mayor parte de las veces son poco favorables a ellos. Por tradición ${ }^{25} y$ por experiencia, el personal del servicio diplomático tiende a favorecer los medios tradicionales de comunicación con sus pares, y a ver a los medios masivos de comunicación como inútiles o peligrosos. Su preferencia es la negociación con colegas extranjeros, y como resultado, tienen una profunda aversión a las técnicas que interfieren con la soberanía de los estados extranjeros.

\section{7. ¿Cuáles son los resultados de la política extranjera?}

Es evidente que la política exterior de los estados lleva a la firma de todo tipo de acuerdos, alianzas - garantías políticas o económicas, a menos que concluya en una situación de aislacionismo político o autarquía económica. Pero para comprender las relaciones internacionales, tal vez sea menos interesante analizar este marco formal que estudiar de cerca la forma en que realmente conviven todos estos sistemas. Los resultados de una política son muy diferentes según el caso. El éxito absoluto es muy raro, y cuando existe en apariencia, es necesario ver si no lleva consigo el germen de una venganza e incluso una destrucción para el beneficiario. En este sentido, la política de Bismarck en 1866 fue más constructiva que en 1871. En contraste con el éxito total, puede darse un fracaso total, de lo cual la política exterior de Mussolini ofrece un buen ejemplo. Pero en la gran mayoría de los casos, una política exterior propone llegar a un compromiso entre dos exigencias aparentemente irreconciliables. Con la excepción de ciertos casos especiales, se puede decir que cualquier frontera, en Europa y en otros lugares, es el resultado de una larga serie de compromisos. Es evidente que el estudio de las fronteras es uno de los métodos más enriquecedores para la comprensión de las relaciones internacionales. Estas separan dos territorios, dos grupos económicos, dos "iconografías" - para usar la expresión de J. Gottmann ${ }^{26}$, es decir, dos formas de concebir la vida-, dos sistemas de educación, de tradición, de instintos colectivos. A través de ellas se

25 Véase SPEIER, Hans, "International Political Communication. Elite vs. Mass" en World Politics, vol. 4, no 3, 1952, p. 307.

${ }^{26}$ Op. cit. Passim. Véase también las obras de ANCEL, Jacques, Manuel géographique de politique européenne, Delagrave, Paris, 1936 y de DION, Roger, Les Frontières de la France, Hachette, Paris, 1947. 
logra un equilibrio cuya naturaleza provisional o definitiva también es muy instructiva. Pero hay muchas otras formas de equilibrio y compromiso además de la frontera. Las relaciones económicas entre dos estados son casi siempre el resultado de un conjunto de compromisos.

\section{8. ¿Cuáles son los objetivos de la política exterior?}

A fin de cuentas, no debemos buscar los objetivos de la política exterior según el ideal que formamos, sino de acuerdo con la realidad de los hechos analizados. Es cierto que tal análisis conduce inevitablemente al interés nacional y a los intereses internacionales. La propia noción de interés nacional es muy compleja, pues es el resultado de la combinación de "intereses generales" de todos los ciudadanos - que es en sí misma fruto de innumerables compromisos ${ }^{27}-y$ "el interés de los gobernantes". En los países democráticos, estas dos formas de interés son casi siempre coincidentes. En los países totalitarios, los líderes persiguen la mayoría de las veces su propio interés, y con ello involucran a sus naciones en políticas de prestigio e imperialismo raramente compatibles con el interés general. En los países subdesarrollados, el interés general es a veces absolutamente incompatible con el interés nacional, pues el primero implica proporcionar los medios para escapar de la hambruna a la mayoría de los habitantes, y ello es a veces solo posible en un estado de dependencia económica y política. De manera general, el interés nacional requiere, antes que nada, lo que puede denominarse como "defensa de la integridad nacional"28, entendiendo las palabras "defensa" e "integridad" en un sentido amplio. La defensa puede ser entendida, en algunos casos, como un paso al frente: por ejemplo, el interés nacional de Alemania implica necesariamente la unión de sus sectores occidental y oriental. La integridad implica no solo la preservación del territorio nacional, sino también el libre desarrollo de ciertas formas de cultura a las que están vinculados los ciudadanos del territorio en cuestión, la posibilidad de mantener y elevar el nivel de vida, etc.

A través del presente epígrafe he intentado confeccionar una lista de las principales preguntas que deben ser formuladas al estudiar el problema de la política exterior. Pero ésta es solo un aspecto de las relaciones internacionales. Resta aún analizar el conjunto de relaciones que ocurren fuera de los gobiernos y representan el accionar de grupos o individuos: aquello que Ilamé anteriormente vida internacional. En relación a ello, no me es posible realizar el mismo trabajo de clasificación que he planteado para la política exterior. Pero es evidente que su interés es extremo: los movimientos migratorios de todo tipo, los problemas de los refugiados, el turismo internacional, los intercambios culturales - a veces completamente independientes de las iniciativas oficiales - los intercambios económicos privados -que, hoy en día, rara vez escapan a los controles estatales-, las misiones religiosas, las peregrinaciones, las reuniones deportivas internacionales. Todo esto entra en el marco de nuestra disciplina.

\section{3.}

Si admitimos que las diversas cuestiones tratadas en el párrafo anterior constituyen el objeto, es decir, el tema de nuestra investigación, no podemos dejar de alarmarnos por la magnitud de la tarea que tenemos entre manos y constatar que apenas la hemos comenzado. Ello hace

\footnotetext{
${ }^{27}$ Véase el análisis abstracto que plantean en torno al interés nacional COOK, Thomas I. y MOOS, Malcolm, "Foreign Policy: The Realism of Idealism" en American Political Science Review, vol. 46, n 2, 1952, pp. 343-356.

${ }^{28}$ Esto surge de las discusiones de una conferencia diplomática celebrada en Clarens en agosto de 1952 por el

"American Friends Service Committee", en el cual tuve el privilegio de participar como consultor.
} 
aún más necesario establecer un método, aunque sea de manera temporal y destinado a mejorar. No obstante ello, gracias a lo realizado hasta el momento nos es posible resumir sus características esenciales.

Según parece, el estudio de las relaciones internacionales debería situarse en tres niveles sucesivos, abordados por diferentes personalidades y grupos, o por las mismas personas de manera coordinada: 1) los estudios específicos o monografías, que deben esforzarse por responder en todos los países de una manera general a una u otra de las preguntas planteadas anteriormente; 2) los estudios de área o estudios generales de los factores de la vida internacional y la política exterior, dedicados al estudio de un determinado territorio o grupos de territorios; 3 ) la teoría general de las relaciones internacionales.

Examinemos uno a uno estos tres niveles de estudio.

\subsection{Las monografías}

De lo dicho anteriormente, resulta indudable que deben realizarse innumerables trabajos históricos, geográficos, legales, antropológicos, sociológicos, psicosociológicos y económicos para comenzar a tener un conocimiento verdaderamente científico en torno a las relaciones internacionales. Esto no significa que no se haya hecho nada. Con respecto a cada una de las preguntas formuladas existen ya estudios en algunos países que tienen a menudo gran valor.

Sobre la influencia de los factores económicos en la política exterior, por ejemplo, hay abundante información en el voluminoso trabajo de Etienne Weill-Raynal, Les Réparations allemandes et la France. Sobre movimientos de población, cómo no mencionar el trabajo del Instituto de Estudios Demográficos o el gran volumen de Victor Purcell, publicado por el "Royal Institute of International Affairs", The Chinese in South East Asia. Estos son, por supuesto, solo ejemplos al azar de una considerable masa de trabajo. Pero, frente a esta documentación ya adquirida, queda un campo mucho más grande por explorar. Esta exploración debe ser la tarea de muchos científicos que trabajen individualmente o en equipos, especializados en disciplinas muy diversas. Como especialistas, ellos persiguen evidentemente sus propios objetivos. Sin embargo, podemos y debemos llamar su atención sobre las necesidades especiales del estudio de las relaciones internacionales, y aumentar con ello su contribución en forma de trabajos útiles. Sería importante que los centros de estudios de Relaciones Internacionales de cada país y, a nivel internacional, la U.N.E.S.C.O. junto a las asociaciones internacionales interesadas, contribuyan a la multiplicación y coordinación de estas monografías. De hecho, en esta etapa de generación de trabajos de referencia, es difícil tener "especialistas en Relaciones Internacionales" capaces de abarcarlo todo. En el artículo anteriormente citado, Frederik S. Dunn muestra que los investigadores se subdividen en varios grupos. Algunos tienen una formación como historiadores diplomáticos, economistas internacionales, juristas internacionales o geógrafos políticos. Otros en sociología, antropología, psicología y psicoanálisis. Es realmente imposible para un solo individuo combinar todo esto. El autor agrega:

En cualquier caso, la investigación en materia de Relaciones Internacionales debe tener en cuenta el hecho de que el campo de conocimiento relativo a los problemas internacionales se ha expandido considerablemente en los últimos años, y ahora incluye todas las ciencias sociales. Si no se puede 
exigir legítimamente que el especialista en política internacional haga el trabajo de todos los demás científicos sociales, sí tiene la responsabilidad especial de ayudar a otros a plantear los problemas reales e interpretar las respuestas que ellos proveen ${ }^{29}$.

\subsection{Los "estudios de área" 30}

\section{Citemos nuevamente a Frederik S. Dunn:}

Un desarrollo de gran interés para la investigación en las Relaciones Internacionales es la rápida expansión de los estudios de área en los últimos años. Esto se debe en gran parte al hecho de que nos dimos cuenta de la inmensa importancia de la influencia de las diversas culturas nacionales en las relaciones entre los pueblos. El objetivo fundamental de los estudios de área es lograr una integración de las diversas técnicas de las ciencias sociales para la comprensión de las sociedades en su conjunto o de unidades geográficas determinadas ${ }^{31}$.

Más precisamente, podemos definir un estudio de área de la siguiente manera: "Se trata de un estudio científico de una región que presenta cierta unidad, y que tiene por objetivo conocer y explicar su lugar exacto (aspecto estático) y su papel (aspecto dinámico) en las relaciones internacionales. Este resultado solo puede ser alcanzado a través del uso sistemático de todas las disciplinas que puedan proporcionar explicaciones válidas".

Por lo tanto, el estudio de área es una nueva etapa en el estudio de las relaciones internacionales y se puede decir que después del esfuerzo analítico que constituyen todas las obras monográficas, es el primer esfuerzo sintético. Síntesis parcial evidentemente, ya que está limitada a una región específica. Síntesis provisoria también, y ello se debe a dos razones: la primera es que cualquier estudio de área realizado en la actualidad solo tiene un conjunto de estudios primarios y monografías insuficientes para llegar a certezas generales; la segunda es que la "situación actual" está, por definición, en constante evolución, y cada cierto tiempo es necesario volver al trabajo y actualizarlo. Además, un estudio de área no es un estudio de geografía regional cuyo propósito es simplemente describir y explicar el paisaje. No es una "guía" descriptiva. Es a la vez científico y sistemático: busca sacar conclusiones sobre las relaciones internacionales de la región estudiada.

Para tener una idea del método propio de los estudios de área -el cual emerge de trabajos ya realizados de este tipo ${ }^{32}$ - es necesario responder a las dos siguientes preguntas. ¿En qué caso puede una región ser sometida a un estudio sistemático? ¿Cómo se pueden

\footnotetext{
${ }^{29}$ DUNN, Frederick S., "The Present Course of International Relations Research" en World Politics, vol. 2, no 1, 1949 , p. 90.

${ }^{30}$ Me gustaría hacer referencia al artículo que escribí en el Bulletin International des Sciences Sociales de la U.N.E.S.C.O. sobre los estudios de área que será publicado a finales de 1952. Me limitaré en esta ocasión a resumir este artículo sin reproducir los elementos bibliográficos que contiene. $N$. del $t .:$ El autor hace referencia al artículo DUROSELLE, Jean B., "Les area studies: problèmes de méthode" en Bulletin International des Sciences Sociales, vol. 4, no 4, 1952, pp. 674-684.

${ }^{31}$ Ibídem, p. 91.

32 Se encontrará una bibliografía crítica en el Bulletin International des Sciences Sociales al cual hice referencia anteriormente (fines de 1952).
} 
determinar las disciplinas cuyo uso es indispensable para el estudio de la región elegida?

A la primera pregunta, podemos responder que no es posible realizar un estudio de área para una fracción de un Estado. La unidad de análisis más pequeña posible es el estado o, si se trata de un territorio dependiente, el territorio que tenga una unidad política. En el caso de un estado federal, como los Estados Unidos y Suiza, solo puede haber un estudio de área si se analiza en su conjunto. Solo cuando un territorio independiente o dependiente tenga una personalidad suficientemente fuerte como para desempeñar un papel en las relaciones internacionales puede ser sujeto de estudio. Esta noción de personalidad es bastante imprecisa, y es por ello necesario resolver por simple sentido común las dificultades que puedan surgir. En el caso de un territorio independiente, se puede admitir que su personalidad existe desde el momento en el cual algunos de sus habitantes desempeñan un rol en el campo de las relaciones internacionales en sentido amplio, que es claramente independiente de la autoridad a la cual están sujetos. Así, mientras podemos considerar un estudio de área en torno a Túnez; es difícil hacer lo mismo con Djibouti. El marco general de la Unión Soviética es preferible al de Ucrania o Turquestán. Por otra parte, también se trata de establecer un marco mínimo de análisis. En este sentido, puede ser ventajoso agrupar varios estados o territorios: el norte de África, medio oriente, el sudeste asiático, América latina, etc. La regla es entonces la siguiente: existe un interés en agrupar múltiples territorios en un estudio de área cuando: a) este grupo presenta cierta unidad étnica, lingüística, histórica, económica, geográfica, etc. de modo que ofrece una buena oportunidad para descubrir datos comunes a quienes constituyen el grupo; b) ninguno de los territorios del grupo tiene una personalidad tal (debido a su larga historia, la importancia de su civilización, su población, etc.) que los efectos del contraste superan las características comunes.

La segunda pregunta: ¿qué disciplinas deberían usarse? implica una respuesta igualmente matizada. Se trata de dar una explicación satisfactoria del rol y el lugar que ocupa un área geográfica determinada en las relaciones internacionales. Toda área geográfica representa un caso concreto. La clave es que el director de la obra, especialista cualificado en Relaciones Internacionales y con una buena formación histórica, geográfica y sociológica, guíe a cada uno de los colaboradores con los cuales trabaja. De lo contrario, corremos el riesgo de ver a cada especialista hacer un excelente estudio en sí mismo, pero poco adaptado al objetivo común. Es cierto que el trabajo debe incluir una parte de la geografía, una de historia y otra de sociología. Cada una de estas tres disciplinas puede prestar diversos servicios: utilizaremos la historia internacional, económica, social, la historia de las instituciones, la de las ideas; la historia demográfica; la geografía física, económica, humana; la sociología de los grupos; la sociología política y religiosa; la psico-sociología; la socio-antropología, etc. Pero, según el caso, también será necesario recurrir a otras disciplinas: antropología, prehistoria, lingüística, zoología, botánica, derecho constitucional, civil, administrativo, etc. La elección es delicada, pero si está bien hecha, los resultados pueden ser de gran interés para lograr el objetivo deseado, que es, repito, un mejor conocimiento científico del lugar y el papel de la región determinada en las relaciones internacionales.

\subsection{La teoría general de las relaciones internacionales}

A primera vista, una teoría general definitiva de las relaciones internacionales no es posible, tal como no es posible una teoría general definitiva de la física, la biología o cualquiera de 
las ciencias sociales. Sin embargo, el progreso del pensamiento humano exige en todos los campos la elaboración de teorías que expliquen una cantidad cada vez mayor de hechos, y los conecten con las hipótesis de trabajo. Estas hipótesis son superadas cuando los hechos que llevan a ellas son superados y el descubrimiento de nuevos hechos las contradicen: el juicio hipotético es, en cierto modo, un juicio de espera, antes de que el hecho pueda ser conocido, probado y explicado. En este caso, es necesario imaginar una teoría más general, que a su vez suponga nuevas hipótesis.

Este enfoque parece ser tan inherente al pensamiento humano, que puede ser aplicado a todos los órdenes de conocimiento. En el estudio de las relaciones internacionales, después del trabajo de análisis de las monografías y la síntesis limitada a un área geográfica determinada, se abre paso a un tercer proceso, que consta de la elaboración de un conjunto de síntesis aún más generales, que constituyen una o más teorías generales.

Básicamente, el trabajo de síntesis es más accesible directamente que los dos procesos precedentes. De hecho, para construir una teoría, basta con pocos hechos y muchas hipótesis. Esto explica el extraordinario desarrollo de lo que comúnmente se llama la "filosofía de la historia". Sus productos constituyen, desde la antigüedad, una enorme biblioteca. La clave es entonces saber distinguir entre los que son válidos y los que no. Los que son válidos, situados en el momento en que fueron escritos, son aquellos que se esfuerzan por tener en cuenta todos los hechos conocidos y dejan de lado aquellos apoyados sobre fundamentos débiles. Con frecuencia se actúa como "filósofo de la historia" porque, para una mente relajada, esto es mucho más sencillo y menos laborioso que la historia. De hecho, es raro que un buen filósofo de la historia no sea primero un buen historiador. Con frecuencia, la mediocridad de este tipo de trabajos proviene del hecho de que no solo se ignoran los hechos ya adquiridos, sino que se deforman para adaptarlos a una teoría general de manera inconsciente - signo de falta de comprensión - o consciente - signo de deshonestidad intelectual-.

En el campo de las Relaciones Internacionales sucede lo mismo. Una teoría válida -ya sea general y que abarque el conjunto de las relaciones, o bien que se limite a un aspecto importante- es aquella que tiene en cuenta todos los hechos esenciales conocidos. Como he dicho antes, esta debe mantenerse sobre el plano científico y objetivo, y no introducir consideraciones normativas que la distorsionen. Por supuesto, todo investigador tiene derecho a tener sus ideas sobre el ideal moral al que la sociedad internacional debería ajustarse, y tiene el derecho de expresarlas, pero no debe mezclarlas con la teoría misma. En el estado actual de nuestro conocimiento del tema, ya es posible escribir obras sintéticas mucho más fácilmente que cuando Maquiavelo escribió El príncipe: la primera teoría verdaderamente científica de las Relaciones Internacionales. Tales "estudios globales"33 no son solo útiles sino necesarios, ya que permiten tener una visión general, que gradualmente se percibirá como exacta o distorsionada.

Por supuesto, su utilidad está estrechamente ligada a la forma en que se relacionan con los hechos. Si se elevan demasiado por encima de ellos y multiplican peligrosamente

\footnotetext{
${ }^{33}$ Para emplear una expresión de Maurice Duverger en una de las sesiones del II Congreso de la Asociación Internacional de Ciencias Políticas en La Haya (septiembre de 1951).
} 
los postulados, pueden ser estimulantes para la mente pero no muy fructíferos para la investigación. Tal sería el caso de las obras de James Burnham: los últimos capítulos de The Managerial Revolution dedicados a las Relaciones Internacionales y The struggle for the world. Mucho más cercanos a los hechos son los libros de Raymond Aron: Le Grand Schisme - Les Guerres en chaîne. Los ejemplos son múltiples. De manera general, los trabajos de síntesis pueden clasificarse en dos categorías: aquellos que toman el conjunto de los problemas de las relaciones internacionales, por ejemplo, Power Politics de Schwarzenberger o An Introduction to World Politics de W. Friedman; y aquellos que solo abordan un aspecto importante, por ejemplo los libros de Hans Kohn sobre nacionalismo, Imperialism and Social Classes de Schumpeter (cuya primera edición data de 1919) o The Origins of Totalitarianism de Hannah Arendt (1951). Sobre temas aún más precisos, citemos como ejemplo la nueva obra de Waldemar Gurian, Bolchevism: an Introduction to Soviet Communism, el trabajo colectivo editado por William Fielding Ogburn, Technology of International Relations (1949), en el cual colaboraron especialistas destacados como W. T. R. Fox y Quincy Wright, o el libro de J. Gottmann, La Politique des Etats et leur géographie (1952).

Es bastante curioso observar que los científicos estadounidenses, que han producido numerosos estudios de área y realizan un notable trabajo analítico en la elaboración de monografías, dejan la tarea de llevar a cabo tales síntesis, en general, a los europeos o a sus colegas formados en Europa ${ }^{34}$.

\section{4.}

Naturalmente, todo esto me conduce a examinar cuáles son las perspectivas abiertas al estudio de las Relaciones Internacionales en Francia ${ }^{35}$. Es interesante observar que esta disciplina no ha tenido en Francia el desarrollo que tiene en los Estados Unidos y Gran Bretaña. Sin embargo, las circunstancias son muy favorables. Francia ocupa un lugar destacado en el estudio del Derecho internacional. La escuela francesa de Historia de las Relaciones Internacionales, con Pierre Renouvin y Maurice Baumont, es universalmente conocida. La escuela geográfica francesa, dirigida por Vidal de la Blanche, Demangeon y Emmanuel de Martonne, fue una de las primeras del mundo, si no la primera.

De hecho, para seguir el esquema propuesto anteriormente, podemos admitir que en la primera y tercera etapa: monografías y síntesis generales, la contribución de los estudiosos franceses está lejos de ser insignificante. Los aportes en geografía y sociología electoral de André Siegfried y François Goguel, en geografía política de André Siegried y Jean Gottmann, en sociología religiosa de Gabriel Le Bras, en demografía e historia demográfica de Alfred Sauvy y Louis Chevalier, etc. ya han acumulado una enorme cantidad de aportes, y las obras en progreso prometen aún muchos más.

\footnotetext{
${ }^{34}$ Véase lo que señala Klaus Knorr ("Theories of Imperialism" en World Politics, vol. 4, no 3, 1952, p. 402): "Una razón para esta notable diferencia radica sin duda en la reticencia tradicional de los científicos sociales angloamericanos a generalizar sobre la causalidad de los acontecimientos históricos. Hay mucho que decir sobre esta falta de voluntad para generalizar fácilmente, contra el irrefrenable entusiasmo con el cual los investigadores europeos construyen sus amplias teorías. Sin embargo, tampoco se puede negar que la tradición europea ha producido teorías de valor excepcional y permanente para la comprensión de los acontecimientos sociales y políticos".

35 Véase en La Science politique contemporaine, el artículo anteriormente citado de P. Renouvin: "La Contribution de la France à l'étude des relations internationales".
} 
La Historia de las Relaciones Internacionales contemporáneas está dando un importante paso adelante con el trabajo de François Le Roy y la publicación de colecciones como Encyclopédie politique ${ }^{36}$ ou Anée politique ${ }^{37}$. También he señalado anteriormente diversas síntesis escritas por investigadores franceses.

Por otro lado, estos autores prestaron muy poca atención a los estudios de área. Uno podría citar el trabajo de A. Basset, L. Bercher, Brunschwig, Initiation à la Tunisie, o incluso en cierta medida Les Fondements de la politique extérieure des Etats-Unis ${ }^{38}$ y otras obras. Pero es sorprendente notar que ningún estudio de área destacable acerca de Francia ha sido escrito por investigadores franceses, y que la política exterior francesa en la actualidad nunca ha sido estudiada científicamente.

A partir de estos datos dispersos, que doy como ejemplo sin pretender que sean completos, se desprende una conclusión evidente: para que Francia ocupe su lugar en una disciplina cuyo interés científico es fundamental y cuyo interés práctico para los diplomáticos franceses es igualmente notorio, es necesario dar un nuevo impulso al esfuerzo de los investigadores franceses.

Dar tal ímpetu debe ser la tarea de las instituciones existentes. Sería arbitrario crear otras nuevas, con todas las implicaciones financieras que ello conllevaría, mientras que el problema esencial es dar a las que existen una conciencia más clara de sus responsabilidades. Basta con enumerar las principales organizaciones que probablemente se muevan resueltamente en esta dirección.

La Fundación Nacional de Ciencias Políticas es sin duda la principal de ellas. Gracias a la flexibilidad de su organización, la variedad de sus actividades y el número de empleados de una amplia gama de especialidades con los que cuenta, es capaz de tomar iniciativas muy fructíferas en el campo de la investigación de las Relaciones Internacionales. Bajo su égida, los investigadores pueden emprender trabajos monográficos; formar grupos para el desarrollo de estudios de área; y publicar trabajos de síntesis. Los numerosos cursos del Instituto de Estudios Políticos de París y algunos Institutos de Estudios Políticos provinciales que se ocupan de las Relaciones Internacionales pueden servir de base para publicaciones originales, y orientar la investigación de los estudiantes. También pueden armonizar y coordinar estos diversos trabajos y colocarlos en un programa integral, teniendo en cuenta las lagunas y las necesidades de la ciencia. Todo esto debe hacerse primero empíricamente y de acuerdo con las posibilidades. La clave es que este impulso sea dado.

El Centro de Política Exterior también puede ser un foco de investigación y estudios en esta área, y su revista Politique Étrangère puede servir como medio de expresión.

Otros institutos más especializados ya contribuyen o pueden hacer una contribución

\footnotetext{
${ }^{36}$ Bajo la dirección de François Le Roy, Maurice Guernier, Georges Rotvand. Edición de Union Française, 4 volúmenes publicados.

${ }^{37}$ Bajo la dirección de André Siegried, Edouard Bonnefous, J.B. Duroselle, colección anual (Presses Universitaires de France).

${ }^{38}$ Cuaderno de la Fundación Nacional de Ciencias Políticas, no 8.
} 
significativa a esta investigación. Mencionemos, entre otros, al Instituto de Estudios Internacionales Avanzados de la Facultad de Derecho de París, que es "antes que nada una escuela especial de Derecho internacional", pero que alberga, en su rico programa anual, muchas lecciones de la disciplina de las Relaciones Internacionales como ha sido definida en este artículo. En la Facultad de Letras de París, es indudablemente posible dar vida a un Instituto de Historia de las Relaciones Internacionales que actualmente existe en los papeles, pero que aún no ha tomado forma. Este Instituto podría proporcionar materiales de valor inestimable en el campo del trabajo histórico. Finalmente, se podrían realizar importantes investigaciones sobre los temas que nos ocupan aquí en varios institutos existentes, como el Instituto Nacional de Estudios Demográficos, el Instituto Científico de Investigación Económica y Social, el Instituto de Ciencias Sociales del Trabajo, el Instituto Francés de Sociología, etc.

Por supuesto, sería muy deseable que se desarrollara una colaboración más activa entre todas las organizaciones que acabo de enumerar, y por supuesto otras que existan. Se trata principalmente de un problema de contactos personales.

¿Carecen estas perspectivas de realismo? Yo no lo creo, por la siguiente razón: en Francia tenemos a los individuos que pueden llevar a cabo estas tareas; pero el problema es en general la falta de dinero. Sin embargo, todo el trabajo por delante no requiere grandes sumas. El dinero serviría principalmente para atraer a investigadores al estudio de las Relaciones Internacionales. Esto podría llevarse a cabo en el campo de los graduados de historia, geografía, filosofía o derecho, o de los egresados de centros de institutos políticos. Muchos de los miembros de estos campos conservan el gusto por la investigación y la profundización de las preguntas. Los extranjeros nos reprochan dar a nuestra élite intelectual una formación de un nivel excepcionalmente alto, para condenarla a las tareas de la educación secundaria donde sería mejor menos ciencia y más pedagogía ${ }^{39}$. A falta de un mayor número de puestos de trabajo en la educación superior - lo cual sería una solución real al problema-, siempre es factible asignarles trabajos científicos que complementen su trabajo profesional. Ello es posible gracias a que la actividad profesional es menos abrumadora en Francia que en cualquier otro lugar, dado a que afortunadamente las horas máximas de trabajo establecidas legalmente tienen un nivel relativamente bajo. De esta manera, estos profesionales estarían involucrados en investigaciones que les proporcionarían una remuneración complementaria. El hecho de que en Francia los historiadores tengan una fuerte formación geográfica y viceversa, nos daría acceso sin grandes esfuerzos a investigadores ya formados, que asimilarían rápidamente la técnica de las Relaciones Internacionales.

Tengamos en cuenta que debido al número de institutos en el extranjero en todas partes del mundo no soviético y al número de profesores que han hecho prácticas en universidades extranjeras, Francia cuenta con un elevado número de personas que tienen o tuvieron contacto directo con la realidad de otros países.

Tal vez sea necesario concluir añadiendo una observación. La cultura francesa, con las cualidades de síntesis que siempre ha demostrado tener y con el gusto por las ideas generales

${ }^{39}$ Véanse las útiles observaciones de PEYRE, "Le Rayonnement de la culture française" en Le Monde, 13 de mayo de 1949, retomado por PADOVER, Saul K., "France in Defeat: Causes and Consequences" en World Politics, vol. 2, no 3, 1950, p. 339. 
que despierta, podría dar una importancia al estudio de las Relaciones Internacionales que apenas existe en los países anglosajones. Es posible que una mayor implicación de Francia con esta nueva disciplina le otorgue más claridad, mejores directrices y un nuevo impulso. Esto no pretende despreciar el inmenso y notable esfuerzo que han realizado en este campo los investigadores anglosajones. Pero, incluso admitiendo que la contribución francesa no sea original, sigue siendo evidente que un país como el nuestro no puede permanecer ajeno al desarrollo de esta disciplina tan enriquecedora, y que es necesario que tanto el interés de nuestra cultura como el de nuestra política aumenten su participación en el trabajo común de los individuos en la búsqueda por entender y explicar sus relaciones a través de las fronteras. 


\section{RELACIONES INTERNACIONALES}

Revista académica cuatrimestral de publicación electrónica Grupo de Estudios de Relaciones Internacionales (GERI) Universidad Autónoma de Madrid, España

www.relacionesinternacionales.info

ISSN 1699 - 3950

ff facebook.com/RelacionesInternacionales

twitter.com/RRInternacional 